\title{
Avaliação da Resposta do Câncer de Mama à Quimioterapia: Papel da Ultra-sonografia e da Dopplerfluxometria
}

\author{
Evaluation of Breast Cancer Response to Chemotherapy: Role of Ultrasound and Doppler
}

Maurício Sena Martins ${ }^{1,2}$, Gustavo Antonio de Souza ${ }^{1}$, Sophie Françoise Mauricette Derchain ${ }^{1}$ Cecilia Maria Roteli-Martins², Valdir Tadini ${ }^{2}$, Angeluce Linhares dos Santos ${ }^{2}$, Silvia Helena Caires de Oliveira ${ }^{2}$

\section{RESUMO}

Objetivo: comparar os tamanhos dos tumores mamários localmente avançados por meio de exame clínico, mamografia, ultra-sonografia e dopplervelocimetria associada à ultra-sonografia, avaliados antes e depois da quimioterapia primária.

Métodos: estudo clínico prospectivo e descritivo. Foram incluidas dezoito mulheres com tumor primário clínico maior ou igual a $50 \mathrm{~mm}$ que foram submetidas à palpação, mamografia, ultrasonografia e dopplervelocimetria, no momento do diagnóstico e após 3 ciclos de quimioterapia. A resposta foi considerada presente quando ocorreu diminuição clínica maior que 50\% e ultra-sonográfica maior que $25 \%$, e ausente quando a redução medida pela clínica fosse inferior $50 \%$ e a ultra-sonográfica menor que 25\%. A análise estatística utilizada foi descritiva com teste de Wilcoxon na comparação dos métodos.

Resultados: em relação ao tamanho dos tumores, o método cuja medida mais se aproximou do exame anatomopatológico foi o exame clinico na pós-quimioterapia, quando comparado com a ultra-sonografia e a mamografia. A mamografia permitiu avaliação em $44 \%$ dos casos, antes da quimioterapia (8/18). Nos casos em que não houve resposta à quimioterapia, a dopplervelocimetria demonstrou aumento significativo do fluxo, com a diminuição dos indices de pulsatilidade e resistência. Nos casos em que houve resposta (9 casos), com redução do tamanho tumoral, a dopplervelocimetria não mostrou alterações significativas em relação aos parâmetros estudados.

Conclusão: o exame clínico mostrou-se como melhor método de avaliação da redução do volume dos tumores tratados com quimioterapia neoadjuvante quando comparado com o exame de ultra-sonografia e a mamografia. A dopplervelocimetria demonstrou que houve aumento do fluxo nos casos com resposta ausente.

PALAVRAS-CHAVE: Mama: câncer. Tratamento neoadjuvante. Quimioterapia. Dopplervelocimetria. Exame clínico.

\section{Introdução}

O câncer de mama corresponde a $9 \%$ das neoplasias malignas em humanos e a $18 \%$ dos cânceres em mulheres no mundo ${ }^{1}$. A proporção de casos desta neoplasia detectados nos estádios III e IV, ou seja, localmente avançados ou metastáticos, oscila entre 10 a $20 \%$ do total, po-

\footnotetext{
${ }^{1}$ Universidade Estadual de Campinas (UNICAMP)

${ }^{2}$ Hospital Leonor Mendes de Barros (São Paulo)

Correspondência: Maurício Sena Martins

Rua Presidente Antônio Cândido, 330 apto 52

05083-060 - São Paulo - SP

Tel: (11) 3259-2359

e-mail: mauriciosena@uol.com.br
}

dendo alcançar até $50 \%$ dos casos novos em alguns países ${ }^{2,3}$. As taxas de sobrevida em cinco anos são de $85 \%$ e $10 \%$ para os estádios I e IV, respectivamente ${ }^{4}$. Já está bem documentado que a cirurgia como tratamento isolado é ineficaz em pacientes com tumores localmente avançados e também em tumores iniciais ${ }^{5}$. Nos últimos anos, estabeleceu-se consenso mundial na utilização e indicação da quimioterapia adjuvante, ou seja, administrada após a retirada cirúrgica da mama ${ }^{6-9}$. A terapia neoadjuvante, inicialmente definida como terapia sistêmica realizada antes do tratamento local, é mais recente ${ }^{10,11}$. Existem diversas propostas de procedimentos para avaliação da regressão tumoral após a quimioterapia neoadjuvante. 
A Organização Mundial da Saúde indica o exame clínico como critério para avaliar a resposta do tumor à terapia neoadjuvante ${ }^{12}$. A utilização de outros exames para determinar a avaliação da resposta à quimioterapia torna-se pertinente, principalmente para melhorar a acurácia do exame clínico a fim de estabelecer normas de conduta, como definir o tipo de cirurgia ou mudança no tratamento. Outros métodos propedêuticos têm sido propostos, associados ao exame clínico, para avaliar a regressão tumoral: mamografia, ultra-sonografia e dopplerfluxometria associada à ultra-sonografia, tomografia contrastada, ressonância magnética com gadolínio (DTPA) e cintilografia mamária com uso de tecnécio $99^{13,14}$.

Estima-se que a mamografia seja o melhor método para rastreamento e diagnóstico do câncer de mama ${ }^{15}$. Entretanto, a mamografia não é considerada muito adequada para avaliação da resposta ao tratamento médico pois superestima a presença de tumor residual após a terapia neoadjuvante quando comparada com o exame clínico ${ }^{16}$.

A ultra-sonografia mamária foi introduzida em 1952 como método diagnóstico ${ }^{17}$. Em carcinomas mamários já diagnosticados, as medidas ultra-sonográficas do tumor têm mostrado maior correlação com a medida do exame anatomopatológico do que aquelas da mamografia ${ }^{13}$.

A dopplervelocimetria colorida associada à ultra-sonografia é método diagnóstico recente que se originou da evolução da ultra-sonografia e posteriormente do Doppler pulsátil ${ }^{18}$. Sua utilização no diagnóstico de imagens veio contribuir para a avaliação funcional, permitindo, com o auxílio das cores, analisar o padrão vascular arterial e veno$\mathrm{so}^{19}$. O desenvolvimento de vasos patológicos em carcinomas devido à resposta a fatores angiogênicos pode levar à diminuição da resistência do fluxo sanguíneo intratumoral; esta característica dos tumores permitiu levantar a hipótese de que a dopplervelocimetria seria capaz de distinguir lesões malignas de benignas ${ }^{20,21}$.

A dopplervelocimetria colorida associada à ultra-sonografia é capaz de avaliar a vascularização tumoral por meio de diversos parâmetros: mensuração da velocidade máxima do fluxo na sístole e sua média, velocidade sistólica mínima, quantificação do número de vasos, soma das velocidades máximas dos vasos e sua média, relação entre sístole e diástole (A/B) máxima e minima e sua média, médias das velocidades e determinação dos índices de resistência e de pulsatilidade e de suas médias, que são expressões matemáticas da resistência dos vasos. Embora haja controvérsias, alguns autores consideram que a dopplervelocimetria colorida associada à ultra-sonografia tem mostrado alta sensibilidade e especificidade no diagnóstico dos tumores mamários ${ }^{22}$. A sensi- bilidade do método em diferenciar tumores benignos de malignos varia entre 91 a $95 \%$, com especificidade de 89 a $97 \%$ e acurácia de $90 \%{ }^{19,23}$.

A mudança na vascularização detectada pela dopplervelocimetria associada à ultra-sonografia pode ser indicador de regressão do tumor, tão bom ou melhor do que a simples mensuração do tumor pela ultra-sonografia ou pela mamografia. A dopplervelocimetria associada à ultra-sonografia poderia ser alternativa mais eficiente à ultrasonografia isolada ou à mamografia. Assim, poderiamos ter novo método que ofereceria vantagens adicionais no estudo da evolução dos tumores mamários submetidos a terapia neoadjuvante. Com isso, seria possivel antecipar precocemente condutas de tratamentos locorregionais nos casos que não estejam respondendo adequadamente à quimioterapia neoadjuvante.

\section{Pacientes e Métodos}

Este foi um estudo clínico prospectivo descritivo comparativo. Foram incluídas neste estudo 18 mulheres portadoras de carcinoma de mama confirmado histologicamente, cujos nódulos tinham mais de $50 \mathrm{~mm}$ no maior diâmetro ( $\mathrm{T}_{3}$ somente). As pacientes foram atendidas no ambulatório de Mastologia do Hospital e Maternidade Leonor Mendes de Barros e avaliadas antes e após a quimioterapia. Como o objetivo do estudo é comparar métodos, foi feita avaliação após a quimioterapia e anteriormente ao tratamento cirúrgico. Foram excluídas as mulheres com idade maior de 70 anos, portadoras de outras neoplasias malignas concomitantes ou prévias ou de processos patológicos que contra-indicassem a quimioterapia primária, portadoras de carcinoma de mama sincrônico ou metasincrônico, as que se encontravam no ciclo gravídico-puerperal e aquelas que não desejaram participar do estudo, além daquelas com manipulações cirúrgicas na mama ou quimioterapias anteriores. Também foram excluídas as mulheres cujo exame histológico não confirmou o diagnóstico de carcinoma mamário, uma vez que as mensurações foram feitas previamente à biópsia.

Após a suspeita clínica de neoplasia maligna e avaliação clínica do tamanho da neoplasia, a paciente foi submetida à mamografia utilizandose um aparelho modelo Senographe 600 da General Electric. O exame foi realizado em duas posições, crânio-caudal e médio-lateral obliqua. A mamografia foi repetida ao final da quimioterapia neoadjuvante. Quando as mulheres haviam sido encaminhadas com a mamografia considerada de boa qualidade, apenas a mamografia pós-quimioterapia foi realizada no Serviço. 
As dimensões dos tumores foram obtidas por mensuração em milimetros, com a medida do maior diâmetro obrigatoriamente igual ou maior que $50 \mathrm{~mm}$, utilizando paquímetro.

A seguir foi realizado o exame de dopplervelocimetria com mapeamento a cores associado à ultra-sonografia em duas etapas, sendo a primeira após o diagnóstico e a segunda após o último ciclo de quimioterapia. O exame foi realizado pelos responsáveis do setor de ultra-sonografia do Hospital Leonor Mendes de Barros. Todos os exames de dopplervelocimetria foram realizados juntamente à ultra-sonografia com um mesmo aparelho (Aloka, modelo SSD-2000).

A avaliação ultra-sonográfica foi feita com transdutor linear de alta frequência - 7,5 MHz com varredura de toda a mama e do tumor nos cortes longitudinais, transversais e radiais, permitindo o dimensionamento da massa através da medida da altura, largura e espessura.

O estudo da vascularização da massa foi feito com o mapeamento a cores para captar os sinais do Doppler. A análise dos sinais do Doppler foi realizada por meio da velocidade de fluxo, soma das velocidades sístólicas dos vasos intratumorais, quantificação do número de vasos intratumorais, indices de resistência, coeficiente A/B e velocidades médias. O exame foi realizado com a paciente em decúbito dorsal horizontal com os braços sob a cabeça, para permitir o melhor estudo dos quadrantes laterais e cauda axilar.

O diagnóstico histológico foi realizado por meio de uma biópsia incisional diferida com remoção de um fragmento de $5 \mathrm{~mm}$ a $10 \mathrm{~mm}$, após a realização dos exames. Nesta amostra, todas as mulheres tiveram o diagnóstico de carcinoma ductal invasivo, com estadiamento clínico III. As mulheres foram submetidas à quimioterapia neoadjuvante, em nível ambulatorial, segundo protocolo assistencial do serviço, com ciclofosfamida na dose de $600 \mathrm{mg} / \mathrm{m}^{2}$ de área de superficie corpórea, adriamicina $(40 \mathrm{mg} /$ $\mathrm{m}^{2}$ de área de superficie corpórea) e 5-fluoracil (600 $\mathrm{mg} / \mathrm{m}^{2}$ de área de superficie corpórea). Esta última foi calculada por meio da obtenção do peso expresso em gramas e da estatura expressa em metros. O intervalo entre os ciclos foi de 21 até 42 dias, dependendo da toxicidade hematológica, totalizando três ciclos, em todos os casos.

Após a quimioterapia neoadjuvante foi realizada a cirurgia indicada para o tratamento da pacientes, novamente sem alteração do protocolo assistencial do serviço. O tamanho clínico do tumor foi medido na sala cirúrgica e a seguir o material foi encaminhado para avaliação macroscópica e microscópica do tamanho do tumor pelo patologista.

Este trabalho foi aprovado pelo Comitê de Ética em Pesquisa (CEP) do Hospital Leonor Men- des de Barros e pelo CEP do Departamento de Tocoginecologia da UNICAMP.

\section{Resultados}

Metade dos casos incluídos neste estudo apresentou resposta ao tratamento neoadjuvante. Não houve casos com progressão tumoral.

Dos métodos empregados para avaliação das dimensões do tumor, observamos que a média dos diâmetros obtida pelo exame clínico foi de 40,7 $\mathrm{mm}$, contra 23,6 $\mathrm{mm}$ pela ultra-sonografia e 34,8 $\mathrm{mm}$ obtidos no exame anatomopatológico. Portanto, o diâmetro médio que mais se aproximou do exame anatomopatológico após a quimioterapia foi o exame clínico, quando comparado com a ultrasonografia e a mamografia.

A mamografia, no entanto, havia permitido a avaliação em apenas $44 \%$ dos casos antes do tratamento (8 casos em 18). Na maioria dos casos a alta densidade mamária não permitiu a mensuração dos tumores (Tabela 1).

Tabela 1 - Tamanho dos tumores em milímetros, segundo o método de avaliação pósquimioterapia neoadjuvante em ordem decrescente de resposta.

\begin{tabular}{|c|c|c|c|c|}
\hline & Mamografia\# & Exame clínico & $\begin{array}{c}\text { Ultra- } \\
\text { sonografia }\end{array}$ & $\begin{array}{l}\text { Exame Anato- } \\
\text { mopatológico }\end{array}$ \\
\hline & NA & 0 & 0 & 45 \\
\hline & NA & 0 & 14 & 0 \\
\hline & 40 & 0 & 17 & 15 \\
\hline & 58 & 10 & 11 & 0 \\
\hline & 30 & 20 & 19 & 20 \\
\hline & 55 & 22 & 25 & 25 \\
\hline & NA & 50 & 40 & 40 \\
\hline & 40 & 20 & 18 & 45 \\
\hline & 55 & 32 & 21 & 36 \\
\hline & NA & 20 & 23 & 25 \\
\hline & 30 & 30 & 24 & 35 \\
\hline & NA & 78 & 35 & 35 \\
\hline & NA & 30 & 21 & 15 \\
\hline & NA & 70 & 0 & 11,5 \\
\hline & 35 & 70 & 40 & 45 \\
\hline & NA & 150 & 55 & 130 \\
\hline & NA & 82 & 37 & 75 \\
\hline & NA & 49 & 25 & 28 \\
\hline Médias & - & 40,7 & 23,6 & 34,8 \\
\hline DP & - & 38,2 & 14,0 & 29,9 \\
\hline
\end{tabular}


Em relação aos parâmetros da dopplervelocimetria descritos, nos nove casos com resposta (redução do tamanho clínico maior que 50\% e ultra- sonográfico maior que $25 \%$ ) não houve diferença significativa quando se comparam os valores pré e pós-tratamento (Tabela 2).

Tabela 2 - Distribuição dos parâmetros da dopplervelocimetria antes e após a quimioterapia, segundo resposta clínica e ultra-sonográfica presente ou ausente.

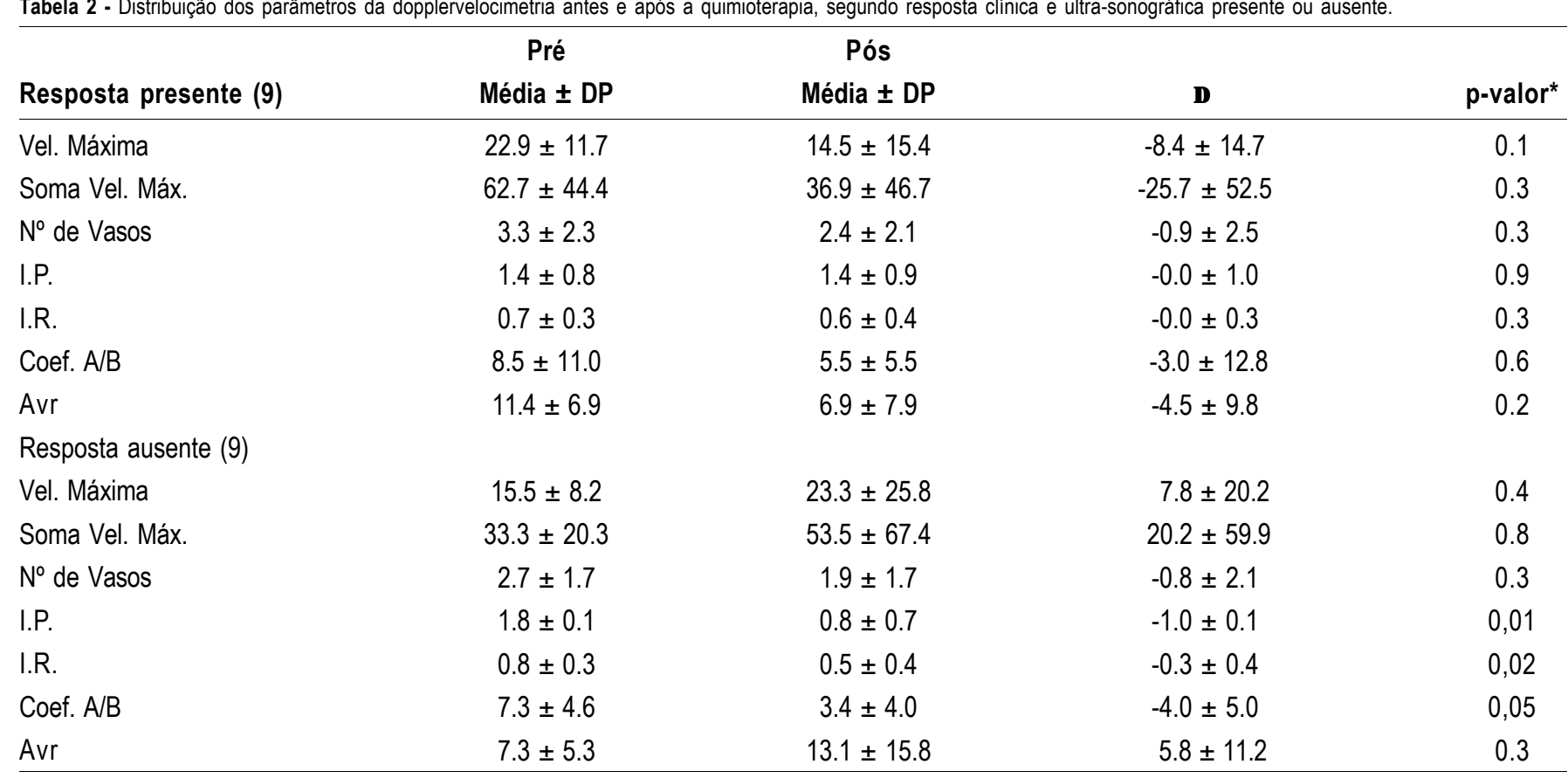

* Teste de Wilcoxon, I.P.= índice de pulsatilidade, IR = índice de residência.

Entre os nove casos que não apresentaram resposta (redução do tamanho clínico $<50 \%$ e ultrasonográfico $<25 \%$ ), o índice de resistência, o índice de pulsatilidade e o coeficiente A/B sofreram redução significativa (valores de $1,8 v s$ s 0,$8 ; 0,8 v s$ 0,5 e 7,3 vs 3,4 , respectivamente.

\section{Discussão}

O objetivo desse estudo foi descrever as características da dopplervelocimetria colorida associada à ultra-sonografia e a mensuração dos tumores antes e depois da quimioterapia neoadjuvante em pacientes com carcinoma de mama localmente avançado e comparar as respostas clínicas, mamográficas e ultra-sonográficas e da dopplervelocimetria associada à ultra-sonografia.

Em relação à avaliação da resposta clínica, mamográfica e ultra-sonográfica, observamos que o exame clínico, seguido pelo ultra-sonográfico, mostrou melhor correlação com o exame anatomopatológico (padrão-ouro). Entretanto, nos poucos casos em que a mamografia possibilitou a mensuração do tumor, este exame apresentou a melhor correlação, mas não pode ser considerado o melhor método, pelas suas limitações.
Entre os parâmetros da dopplervelocimetria colorida associada à ultra-sonografia, foi observado que o índice de resistência, o índice de pulsatilidade e o coeficiente A/B apresentaram diferença significativa antes e depois da quimioterapia no grupo sem resposta. Após a comparação das pacientes que apresentaram resposta com aquelas que não apresentaram, observou-se que a velocidade média poderia ser útil nessa avaliação. Além disso, este estudo sugere que se pode predizer a resposta pela velocidade média e possivelmente pela velocidade máxima do vaso com maior fluxo.

Constatamos diminuição da vascularização do tumor no grupo de mulheres com resposta, ao passo que as mulheres com ausência de resposta apresentaram aumento de vascularização.

Os resultados deste estudo sugerem que a dopplervelocimetria colorida associada à ultra-sonografia pode ser útil na avaliação da resposta à quimioterapia. Os resultados coincidem com a sugestão de alguns autores como Cosgrove et al. ${ }^{19}$, que observaram que o melhor parâmetro da dopplervelocimetria colorida para avaliar massas mamárias é o índice de resistência. Isso é explicado pela não-dependência do ângulo de incidência do som, como observado por Madjar et al. ${ }^{24}$. 
Estes autores consideraram a velocidade máxima, a soma das velocidades e o número de vasos como sendo os parâmetros de eleição para avaliação de tumores malignos da mama, mas tais parâmetros dependem do ângulo de insonação.

Além disso, quando se analisam a soma das velocidades máximas e o número de vasos, existe a possibilidade de se estar avaliando o mesmo vaso em pontos diferentes, fato este explicado pela tortuosidade e trajetos irregulares da neovascularização tumoral, hiperdimensionando seus valores.

Entretanto, deve-se ressaltar que o melhor exame para avaliar a resposta à quimioterapia continua sendo o clínico. A mamografia e os demais parâmetros da dopplervelocimetria associada à ultra-sonografia não melhoraram os resultados obtidos pelo exame clínico e a ultra-sonografia nesta amostra.

Os resultados deste estudo não coincidem com os resultados encontrados por Fornage et al. ${ }^{25}$ e Bassett e Kimme-Smith ${ }^{13}$, que demonstraram boa concordância do tamanho do tumor pelo exame ultra-sonográfico com a peça anatômica. Isto pode ser explicado pelos efeitos da quimioterapia nestes tumores. O exame ultra-sonográfico mostrou-se, portanto, inadequado para ser utilizado como padrão-ouro para a resposta tumoral à terapia. Somente foi possivel a avaliação de todos os casos por meio do exame clínico e da ultra-sonografia. Esses resultados contrastaram com a resposta da mamografia, que apesar de ter apresentado o melhor índice de correlação, conseguiu avaliar apenas $44 \%$ dos casos. Tais evidências levamnos a refletir que um método que é incontestável no diagnóstico do câncer de mama, como a mamografia, pode não ter muita validade na avaliação da resposta tumoral à quimioterapia.

O primeiro ponto que pode explicar as limitações da mamografia para avaliar o tamanho tumoral é que as incidências obtidas pelo método são magnificadas pela distância tumor-filme ${ }^{25}$. Já a ultra-sonografia apresenta imagem livre de magnificação ou, em outros termos, imagem próxima dos valores reais. O segundo ponto que ajuda a explicar as limitações da mamografia para a avaliação da resposta tumoral é o número de incidências utilizadas; se o maior diâmetro do tumor se apresentar em outra projeção, pode não estar sendo representado o seu maior diâmetro. A ultrasonografia tem a vantagem, sob este aspecto, de fornecer o volume tumoral, o que pode ser, em termos de resposta, uma melhor aproximação com as medidas do exame anatomopatológico. O terceiro ponto relacionado às limitações da mamografia está representado pela dificuldade em definir com precisão as bordas dos tumores. Entretanto, Bassett e Kimme-Smith ${ }^{13}$ sugeriram que o exame ultra-sonográfico tem também limitações para a demarcação das bordas tumorais. Neste sentido, é o exame clínico o que define com maior precisão as bordas da lesão.

Outro aspecto interessante está na relação entre os resultados das avaliações clínicas, mamográficas e ultra-sonográficas pós-quimioterapia de indução e o resultado anatomopatológico. Nesta amostra observou-se esta associação em alguns casos, o que é explicado pelas grandes dimensões dos tumores.

Entendemos que a proposta de utilizar o índice de resistência, pulsatilidade, coeficiente A/ $B$ e velocidade média medida pela dopplervelocimetria colorida associada à ultra-sonografia, como indicadores de resposta, é nova na literatura e merece destaque, mas será preciso acumular casuística mais extensa nesta linha de pesquisa e a confirmação por outros autores, para tornar útil a sua aplicação clínica.

Nossa casuística, pelo seu tamanho, não permitiu avaliar se as características da vascularização do tumor de acordo com a dopplervelocimetria colorida poderia servir para o prognóstico dessa resposta.

Em conclusão, foi possivel com este estudo verificar que o exame clínico mostrou-se como melhor método de avaliação da redução do volume tumoral à quimioterapia neoadjuvante quando comparado com a ultra-sonografia e a mamografia. A dopplervelocimetria demonstrou que houve aumento do fluxo nos casos com resposta ausente, sugerindo que a maior angiogênese nos tumores pode ser um fator associado a pior resposta à quimioterapia.

\section{ABSTRACT}

Purpose: to compare the size of locally advanced breast tumors through clinical examination, mammography, and ultrasound, and describe the parameters of color Doppler ultrasound evaluated before and after primary chemotherapy. Methods: it was a prospective and descriptive clinical study. Eighteen women with the diagnosis of breast carcinoma, with clinical primary tumors of $50 \mathrm{~mm}$ or larger, were submitted to palpation, mammography, ultrasound and color Doppler ultrasound at the moment of diagnosis and after 3 cycles of chemotherapy. The response was considered present when there was a clinical decrease of $50 \%$ or more and an ultrasonographic decrease of $25 \%$ or more, and absent when there was a clinical decrease less than 50\% and ultrasonographic decrease less than 25\%. A descriptive statistical analysis and Wilcoxon test were used.

Results: regarding the size of the tumors, the method whose 
results were closer to pathological examination was the clinical examination after chemotherapy, when compared with sizes on ultrasound and mammography. The mammography helped to evaluate $44 \%$ of the cases before chemotherapy (8) 18). In the cases where the response was absent, the Doppler showed a significant increase of the flow and a decrease of resistance and pulsatility index. In the cases where the response was present (9 cases), the Doppler did not show any significant change regarding the studied parameters.

Conclusion: clinical examination was the most efficient method to evaluate the decrease of the volume of tumors submitted to neoadjuvant chemotherapy when compared with ultrasound and mammographic examinations. Doppler showed increase of the flow in the cases of absent response.

KEY WORDS: Breast: neoplasms. Neoadjuvant treatment. Chemotherapy. Doppler. Clinical examination.

\section{Referências}

1. Parkin DM, Laara E, Muir CS. Estimates of the worldwide frequency of sixteen major cancers in 1980. Int J Cancer 1988; 41:184-97.

2. Hortobagyi GN, Blumenschein GR, Spanos W, et al. Multimodal treatment of locoregionally advanced breast cancer. Cancer 1983; 51:763-8.

3. Schwartz GF, Cantor RI, Biermann WA. Neoadjuvant chemotherapy before definitive treatment for stage III carcinoma of the breast. Arch Surg 1987; 122:1430-4.

4. Pinotti JA, Teixeira LC. Cancer de mama epidemiologia e fatores de risco. In: Halbe HW, editor. Tratado de Ginecologia. $1^{\mathrm{a}}$ ed. São Paulo: Roca; 1987. p.1412-4.

5. Haagensen CD. Clinical classification of the stage of advancement of breast carcinoma. In: Haagensen $\mathrm{CD}$, editor. Diseases of the Breast. $3^{\text {rd }}$ ed. Philadelphia: Saunders; 1986. p.851-63.

6. Fisher B, Carbone P, Economou SG, et al. 1Phenylalanine mustard (L-PAM) in management of primary breast cancer: a report of early findings. $\mathrm{N}$ Engl J Med 1975; 292:117-22.

7. Nissen-Meyer R, Kjellgren K, Malmio K, Mansson $B$, Norin T. Surgical adjuvant chemotherapy: results with one short course with cyclophosphamide after mastectomy for breast cancer. Cancer 1978; 41:2088-98

8. Fisher B, Bauer M, Wickerman L, et al. Relation of number of positive axillary nodes to the prognosis of patients with primary breast cancer: an NSABD update. Cancer 1983; 52:1551-7.

9. Auclerc G, Borel C, Khayat D, Soubrane C, Weil M. La chimiothérapie première dans le traitement du cancer du sein. Ann Chir Plast Esthét 1992; 37:663-9.
10.Frank JL, McClish DK, Dawson KS, Bear HD. Stage III breast cancer: is neoadjuvant chemoterapy always necessary? J Surg Oncol 1992; 49:220-5.

11.Trimble EL, Ungerleider RS, Abrams JA, et al. Neoadjuvant therapy in cancer treatment. Cancer 1993; 72 (Suppl 11):3515-24.

12.Hayward JL, Meakin JW, Stewart HJ. Assessment of response and recurrence in breast cancer. Semin Oncol 1978; 5:445-9.

13.Bassett LW, Kimme-Smith C. Breast sonography: review article. AJR Am J Roentgenol 1991; 156:449-55.

14.Moskovic EC, Mansi JL, King DM, Murch CR, Smith IE. Mammography in the assessment of response to medical treatment of large primary breast cancer. Clin Radiol 1993; 47:339-44.

15.Ennis JT. Radiologia diagnóstica nas doenças da mama. In: Bland KI, Copeland EN, editores. A Mama: tratamento compreensivo das doenças benignas e malignas. $1^{a}$ ed. São Paulo: Manole; 1994. p.489-531.

16.Evans WP. Breast masses. Appropriate evaluation. Radiol Clin North Am 1995; 33:1085-1088.

17.Wild JJ, Neal D. The use of high frequency ultrasonic waves for detecting changes of texture in the living tissue. Lancet 1951; 260:655-7.

18.Price RR, Merritt CRB. Principles and instrumentation. In: Fleischer AC, Emerson DS, editors. Color Doppler Sonography in Obstetrics and Gynecology. $1^{\text {st }}$ ed. New York: Churchill-Livingstone; 1993. p.1-16.

19. Cosgrove DO, Bamber JC, Davey JB, McKinna JA, Sinnett HD. Color doppler signals from breast tumors. Radiology 1990; 176:175-80.

20.Folkman J, Merler E, Abernathy A, Williams G. Isolation of a tumor factor responsible for angiogenesis. J Exp Med 1971; 133:275-88.

21.Folkman J. What is the evidence that tumors are angiogenesis dependent? J Natl Cancer Inst 1990; 82:4-6.

22.Schoenberger SG, Sutherland CM, Robinson AE. Breast neoplasms: duplex sonographic imaging as an adjunct in diagnosis. Radiology 1988; 168:665-8.

23.Britton PD, Coulden RA. The use of duplex Doppler ultrasound in the diagnosis of breast cancer. Clin Radiol 1990; 42:399-401.

24.Madjar H, Prompeler HJ, Sauerbrei W, Wolfarth R, Pfleiderer A. Color Doppler flow criteria of breast lesions. Ultrasound Med Biol 1994; 20:849-58.

25.Fornage BD, Toubas O, Morel M. Clinical, mammographic, and sonographic determination of preoperative breast cancer size. Cancer 1987; 60:765-71. 